\title{
Smoking is not associated with recurrence and retreatment of intracranial aneurysms after endovascular coiling
}

\author{
Waleed Brinjikji, MD, ${ }^{1}$ Ravi K. Lingineni, MPH, ${ }^{2}$ Chris N. Gu, MD, ${ }^{1}$ Giuseppe Lanzino, MD, $, 1,3$ \\ Harry J. Cloft, MD, PhD, ${ }^{1,3}$ Lauren Ulsh, ${ }^{1}$ Kristen Koeller, ${ }^{1}$ and David F. Kallmes, MD1,3 \\ Departments of ${ }^{1}$ Radiology, ${ }^{2}$ Biomedical Statistics and Informatics, and ${ }^{3}$ Neurosurgery, Mayo Clinic, Rochester, Minnesota
}

\begin{abstract}
OBJECT Tobacco smoking is one of the most important risk factors for the formation of intracranial aneurysms and for aneurysmal subarachnoid hemorrhages. Smoking has also been suggested to contribute to the recurrence of aneurysms after endovascular coiling. To improve the understanding of the impact of smoking on long-term outcomes after coil embolization of intracranial aneurysms, the authors studied a consecutive contemporary series of patients treated at their institution. The aims of this study were to determine whether smoking is an independent risk factor for aneurysm recurrence and retreatment after endovascular coiling.
\end{abstract}

METHODS All patients who had received an intrasaccular coil embolization of an intracranial aneurysm, who had undergone a follow-up imaging exam at least 6 months later, and whose smoking history had been recorded from January 2005 through December 2012 were included in this study. Patients were stratified according to smoking status into 3 groups: 1) never a smoker, 2) current smoker (smoked at the time of treatment), and 3) former smoker (quit smoking before treatment). The 2 primary outcomes studied were aneurysm recurrence and aneurysm retreatment after treatment for endovascular aneurysms. Kruskal-Wallis and chi-square tests were used to test statistical significance of differences in the rates of aneurysm recurrence, retreatment, or of both among the 3 groups. A multivariate logistic regression analysis controlling for smoking status and for several characteristics of the aneurysm was also performed.

RESULTS In total, 384 patients with a combined total of 411 aneurysms were included in this study. The aneurysm recurrence rate was not significantly associated with smoking: both former smokers (OR 1.00, 95\% $\mathrm{Cl} 0.61-1.65 ; p=0.99)$ and current smokers (OR 0.58, 95\% $\mathrm{Cl} 0.31-1.09 ; p=0.09)$ had odds of recurrence that were similar to those who were never smokers. Former smokers (OR $0.78,95 \% \mathrm{Cl} 0.46-1.35 ; p=0.38)$ had odds of retreatment similar to those of never smokers, and current smokers had a lower odds of undergoing retreatment (OR 0.44, 95\% $\mathrm{Cl} 0.21-0.91 ; p=0.03)$ than never smokers. Moreover, an analysis adjusting for aneurysm rupture, diameter, and initial occlusion showed that former smokers (OR 0.65, 95\% Cl 0.33-1.28; $p=0.21$ ) and current smokers (OR 1.04, 95\% Cl 0.60-1.81; $p=0.88$ ) had odds of aneurysm recurrence similar to those who were never smokers. Adjusting the analysis for aneurysm rupture, diameter, and occlusion showed that both former smokers (OR 0.49, 95\% Cl 0.23-1.05; $p=0.07$ ) and current smokers (OR 0.82, $95 \% \mathrm{Cl} 0.46-1.46 ; p=0.50$ ) had odds of retreatment similar to those of patients who were never smokers.

CONCLUSIONS The results show that smoking was not an independent risk factor for aneurysm recurrence and aneurysm retreatment among patients receiving endovascular treatment for intracranial aneurysms at the authors' institution. Nonetheless, patients with intracranial aneurysms should continue to be counseled about the risks of tobacco smoking. http://thejns.org/doi/abs/10.3171/2014.10.JNS141035

KEY WORDS aneurysm; endovascular coiling; smoking; vascular disorders

$\mathrm{T}$ obacco smoking is one of the most important risk factors for intracranial aneurysm formation and for an aneurysmal subarachnoid hemorrhage (aSAH). ${ }^{2,6-9,12,13}$ Previous studies have shown that smoking cigarettes increases the risk for an aSAH up to 6-fold when compared with the risk in the general population., ${ }^{2,12}$ Results from one study have indicated that smoking also represents a risk factor for the recurrence of an aneurysm after its treatment with endovascular coiling..$^{11}$ To gain a better understanding of the impact of smoking on long-

ABBREVIATION aSAH = aneurysmal subarachnoid hemorrhage.

SUBMITTED May 9, 2014. ACCEPTED October 7, 2014.

INCLUDE WHEN CITING Published online November 7, 2014; DOI: 10.3171/2014.10.JNS141035.

DISCLOSURE Dr. Lanzino is a consultant for ev3/Covidien and for Codman/Johnson and Johnson. 
term outcomes after endovascular embolization of intracranial aneurysms, we studied these outcomes among a consecutive contemporary series of patients treated at our institution. The goal of this study was to determine whether smoking is an independent risk factor for aneurysm recurrence and retreatment after endovascular treatment. We hypothesized that endovascular treatments of aneurysms would result in a higher rate of aneurysm recurrence, retreatment, or of both among patients who smoked or had a history of smoking than among patients who never smoked.

\section{Methods}

\section{Patients and Study Design}

After receiving approval from the internal review board, we conducted a retrospective chart review of all patients who had received endovascular treatment of intracranial aneurysms from January 2005 through December 2012. The inclusion criteria in this study were that patients had received intrasaccular coil embolization of an intracranial aneurysm, had undergone a follow-up imaging exam at least 6 months later, and had a record of a smoking history. Patients who had undergone a planned and staged multisession embolization were excluded. A retreatment was considered planned and staged if the treating team mentioned in the operative note or followup clinical notes that further treatment was recommended to improve aneurysm occlusion. All patients underwent at least 1 follow-up digital subtraction angiography exam or MR angiography exam.

Once the patients were selected, their data were collected, including demographic characteristics (sex, age, and smoking history), aneurysm characteristics (rupture status, maximum diameter, and location), treatment characteristics (type of treatment and retreatment), initial aneurysm occlusion, use of adjunctive devices such as a stent or balloon, length of follow-up, and any aneurysm occlusion at follow-up.

Smoking status was determined from each patient's medical history questionnaire obtained before or during the treatment. The questionnaire asked the following questions: 1) "Do you currently smoke?" and 2) "Have you ever smoked? If so, how long ago did you quit smoking?" Smoking status was then stratified into 3 groups: 1) never a smoker, 2) current smoker (smoked at the time of treatment), and 3) former smoker (quit smoking before treatment). The 2 primary outcomes of this study were aneurysm recurrence and aneurysm retreatment after an endovascular aneurysm treatment. Initial aneurysm occlusion was categorized according to the Raymond scale into the following: 1) residual aneurysm, 2) residual neck, and 3) complete occlusion. Follow-up aneurysm occlusion status was categorized into the following 2 outcomes: 1 ) stable occlusion and 2) aneurysm recurrence defined as coil compaction, recanalization, or both.

\section{Statistical Analysis}

Continuous variables are presented as mean \pm SD and categorical variables as frequency (as percentage). Kruskal-Wallis and chi-square tests were used to test statistical significance in aneurysm recurrence or retreatment ac- cording to differences in patient characteristics. A univariate logistic regression analysis was performed to test for an association of the primary outcomes, aneurysm recurrence or retreatment, with age, sex, smoking status, aneurysm characteristics (rupture status, initial occlusion, location, and maximum diameter), adjunctive device usage, and length of follow-up. A multivariate logistic regression analysis was then performed, adjusting for the differences in the patient groups with primary outcomes as the dependent variable and smoking status and variables that were significant at the univariate level as the independent variables. All statistical analysis was performed using SAS version 9.3 .

\section{Results}

\section{Patient and Aneurysm Characteristics}

In this study, 384 patients who had a combined total of 411 aneurysms were included. Thirty-nine patients with a combined total of 44 aneurysms treated with flow diversion and 5 patients with 1 aneurysm each who underwent a planned, staged treatment were excluded from this study. Of the patients included, 23 had 2 treated aneurysms, and 2 patients had 3 treated aneurysms. Overall, 106 patients with 111 aneurysms $(27.0 \%)$ had recurrence of an aneurysm, and of these patients, 78 with 80 aneurysms (19.5\%) underwent retreatment for the aneurysm. In the remaining patients with recurrent aneurysms, the recurrence was not considered of clinical significance and observation only was recommended.

The demographics, main aneurysm characteristics, and immediate angiographic results for the 3 patient groups are summarized in Table 1. The mean age of all patients was $61 \pm 12.2$ years, and the mean length of follow-up was $2.4 \pm 2.3$ years. An analysis by smoking status showed that 172 patients with 193 aneurysms $(47.0 \%)$ were current smokers, 95 patients with 99 aneurysms (24.1\%) were former smokers, and 117 patients with 119 aneurysms $(29.0 \%)$ were never smokers. In total, 272 treated aneurysms (66.2\%) were unruptured and 139 (33.8\%) were ruptured, and the mean diameter of all aneurysms was $7.5 \pm$ $4.3 \mathrm{~mm}$. In total, 186 aneurysms (45.3\%) were completely occluded on initial treatment, $185(45.0 \%)$ had a residual neck, and $40(9.7 \%)$ had a residual aneurysm and were not occluded. In a subset of 94 aneurysms (22.9\%), an adjunctive device was used, with 93 of these aneurysms (98.9\%) receiving balloon-assisted coiling and 1 aneurysm (1.1\%) receiving stent-assisted coiling.

With a mean age of $65.2 \pm 11.3$ years, former smokers were significantly older than those who were never smokers $(61.1 \pm 14.4$ years) and were current smokers $(58.5 \pm$ 10.3 years) ( $<<0.001)$. The aneurysm diameter was not significantly different among the groups $(p=0.35)$, with mean diameters of $7.8 \pm 5.2 \mathrm{~mm}$ for those who were never smokers, $7.6 \pm 3.5 \mathrm{~mm}$ for those who were former smokers, and $7.3 \pm 3.9 \mathrm{~mm}$ for those who were current smokers. The 3 groups differed in the proportion of those having ruptured aneurysms: $37.8 \%$ of current smokers (73 aneurysms), $36.1 \%$ who were never smokers (43 aneurysms), and $23.2 \%$ of former smokers (23 aneurysms) had ruptured aneurysms, and a chi-squared test detected a statisti- 
TABLE 1. Patient demographics and aneurysm characteristics by smoking status*

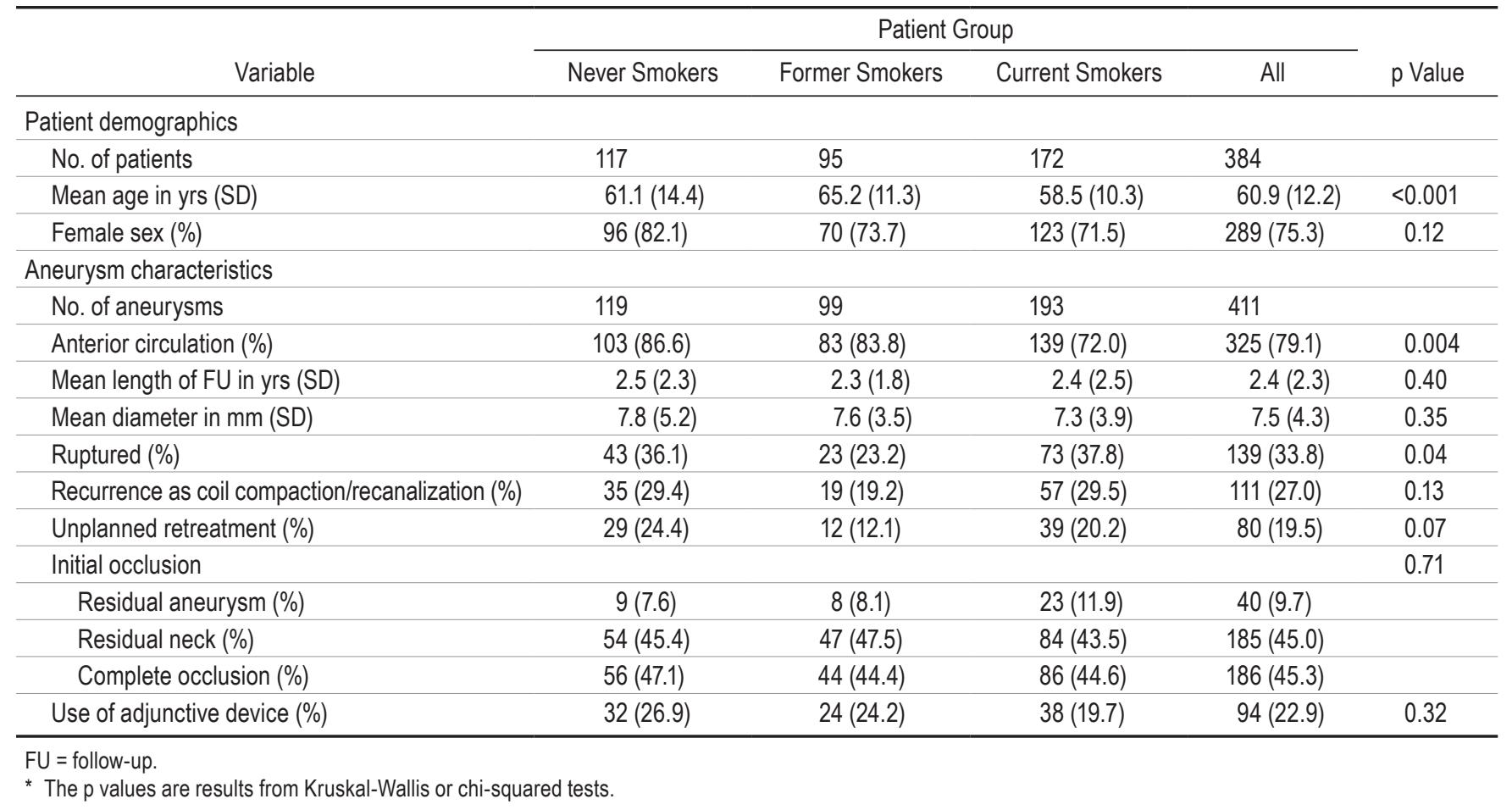

cally significant difference across the 3 groups $(\mathrm{p}=0.04)$. The 3 groups did not significantly differ in the initial status of aneurysm occlusion $(p=0.71)$.

\section{Univariate Analysis of Aneurysm Recurrence}

Results of the univariate analysis of aneurysm recurrence are shown in Table 2 . The aneurysm recurrence rate was not significantly associated with smoking: aneurysms in both former smokers (OR 1.00, 95\% CI 0.61-1.65; p = 0.99) and current smokers (OR 0.58, 95\% CI 0.31-1.09; $\mathrm{p}=$ 0.09 ) had similar odds of recurrence when compared with aneurysm recurrence in patient who were never smokers. Among unruptured aneurysms, the recurrence rate was $23.7 \%$ (18 aneurysms) for the aneurysms in patient who were never smokers, $17.1 \%$ (13 aneurysms) for those in former smokers, and $23.3 \%$ (28 aneurysms) for those in current smokers $(\mathrm{p}=0.62)$. Among ruptured aneurysms, the recurrence rate was $39.5 \%$ (17 aneurysms) for the aneurysms in patients who were never smokers, $26.1 \%$ (6 aneurysms) for those in former smokers, and 39.7\% (19 aneurysms) for those in current smokers $(\mathrm{p}=0.60)$.

The size of the aneurysm diameter was associated with aneurysm recurrence rate; a 1-mm increase in diameter was associated with a significantly $(\mathrm{p}<0.001)$ higher odds in the rate of aneurysm recurrence (OR 1.16, 95\% CI 1.10-1.22). Ruptured aneurysms had a significantly higher odds of recurrence (OR 2.15, 95\% CI 1.38-3.37; $\mathrm{p}=0.001$ ) compared with unruptured aneurysms. Aneurysms with a residual aneurysm (OR 2.42, 95\% CI 1.16-5.04; $\mathrm{p}=0.02$ ) or a residual neck (OR 1.88, 95\% CI 1.17-3.01; $\mathrm{p}=0.01$ ) on initial treatment had significantly higher odds of recurrence compared with those with complete occlusion.

\section{Univariate Analysis of Aneurysm Retreatment}

Table 3 presents the results of univariate analysis of aneurysm retreatment in the 3 groups. Compared with patients who were never smokers, former smokers had similar odds of undergoing retreatment (OR $0.78,95 \%$ CI $0.46-1.35 ; \mathrm{p}=0.38$ ), and current smokers had significantly lower odds of undergoing retreatment (OR $0.44,95 \% \mathrm{CI}$ $0.21-0.91 ; p=0.03)$. Among unruptured aneurysms, the retreatment rate was $19.7 \%$ (18 aneurysms) for the aneurysms in patients who were never smokers, $10.5 \%$ (8 aneurysms) for those in former smokers, and $15.8 \%$ (19 aneurysms) for those in current smokers $(\mathrm{p}=0.34)$. Among ruptured aneurysms, the retreatment rate was $32.6 \%$ (14 aneurysms) for the aneurysms in patients who were never smokers, $17.4 \%$ (4 aneurysms) for those in former smokers, and 27.4\% (20 aneurysms) for those in current smokers $(\mathrm{p}=0.49)$.

The size of the aneurysm diameter was associated with aneurysm retreatment rate; a 1-mm increase in diameter was associated with a significantly $(\mathrm{p}<0.001)$ increased odds of aneurysm retreatment (OR 1.13, 95\% CI 1.071.19). Ruptured aneurysms had a significantly higher odds of retreatment (OR 2.06, 95\% CI 1.25-3.38; p = 0.004) compared with unruptured aneurysms. Aneurysms with a residual aneurysm (OR 1.61, 95\% CI 0.70-3.71; $\mathrm{p}=0.26$ ) or residual neck (OR 1.58, 95\% CI 0.94-2.67; $\mathrm{p}=0.09$ ) on initial treatment were not significantly associated with aneurysm retreatment.

\section{Multivariate Analysis}

The results of multivariate analyses indicating the likelihood of aneurysm recurrence or retreatment among the 3 patient groups and for various patient and aneurysm characteristics are shown in Tables 2 and 3. When adjusting for 
TABLE 2. Results of unadjusted and adjusted univariate analyses indicating the likelihood of aneurysm recurrence*

\begin{tabular}{|c|c|c|c|c|}
\hline \multirow[b]{2}{*}{ Variable } & \multicolumn{2}{|c|}{ Unadjusted Analysis } & \multicolumn{2}{|c|}{ Adjusted Analysis } \\
\hline & OR $(95 \% \mathrm{Cl})$ & $p$ Value & OR $(95 \% \mathrm{Cl})$ & $\mathrm{p}$ Value \\
\hline Age & $0.99(0.98-1.01)$ & 0.456 & - & - \\
\hline Female sex & $0.67(0.41-1.08)$ & 0.102 & - & - \\
\hline Anterior circulation aneurysm & $0.62(0.37-1.03)$ & 0.064 & - & - \\
\hline Length of FU & $1.06(0.97-1.16)$ & 0.211 & - & - \\
\hline Aneurysm diameter & $1.16 \dagger(1.10-1.22)$ & $<0.001 \ddagger$ & $1.18(1.11-1.26)$ & $<0.001$ \\
\hline Ruptured aneurysm & $2.15(1.38-3.37)$ & $0.001 \ddagger$ & $2.92(1.76-4.83)$ & $<0.001$ \\
\hline \multicolumn{5}{|l|}{ Smoking $\mathrm{Hx}$} \\
\hline Never smoker & Reference & NA & Reference & NA \\
\hline Former smoker & $0.58(0.31-1.09)$ & 0.089 & $0.65(0.33-1.28)$ & 0.214 \\
\hline Current smoker & $1.00(0.61-1.65)$ & 0.992 & $1.04(0.60-1.81)$ & 0.878 \\
\hline \multicolumn{5}{|l|}{ Initial aneurysm occlusion } \\
\hline Residual aneurysm & $2.42(1.16-5.04)$ & $0.018 \ddagger$ & $3.13(1.37-7.13)$ & 0.007 \\
\hline Residual neck & $1.88(1.17-3.01)$ & $0.009 \ddagger$ & $1.74(1.04-2.90)$ & 0.034 \\
\hline Complete occlusion & Reference & NA & Reference & NA \\
\hline Use of adjunctive device & $0.74(0.43-1.26)$ & 0.265 & - & - \\
\hline
\end{tabular}

$\mathrm{Hx}=$ history; $\mathrm{NA}=$ not applicable; $-=$ not determined.

* Only smoking history and variables that were statistically significant in the unadjusted analysis were entered into the adjusted analysis.

$\dagger$ This value indicates the OR for every 1 -mm increase in aneurysm diameter.

¥ These $p$ values were identified as being statistically significant in the unadjusted analysis.

the rupture status and diameter of an aneurysm and for an occurrence of initial aneurysm occlusion, the multivariate analysis indicated that former smokers (OR $0.65,95 \%$ CI $0.33-1.28 ; \mathrm{p}=0.21)$ and current smokers $(\mathrm{OR} 1.04$, 95\% CI $0.60-1.81 ; \mathrm{p}=0.88$ ) had odds of aneurysm recurrence similar to those who were never smokers (Table 2). A larger aneurysm diameter was significantly associated with aneurysm recurrence (OR 1.18, 95\% CI 1.11-1.26; $\mathrm{p}<0.001$ ), and ruptured aneurysms had a significantly higher rate of recurrence than unruptured aneurysms (OR $2.92,95 \%$ CI 1.76-4.83; $\mathrm{p}<0.001)$. Initial aneurysm occlusion with a residual neck (OR 1.74, 95\% CI 1.04-2.90; $\mathrm{p}=0.03)$ and a residual aneurysm on initial treatment (OR $3.13,95 \%$ CI 1.37-7.13; $p=0.01$ ) resulted in a higher likelihood of aneurysm recurrence compared with completely occluded aneurysms.

TABLE 3. Results of unadjusted and adjusted analyses indicating the likelihood of aneurysm retreatment*

\begin{tabular}{|c|c|c|c|c|}
\hline \multirow[b]{2}{*}{ Variable } & \multicolumn{2}{|c|}{ Unadjusted Analysis } & \multicolumn{2}{|c|}{ Adjusted Analysis } \\
\hline & OR $(95 \% \mathrm{Cl})$ & p Value & OR (95\% Cl) & $\mathrm{p}$ Value \\
\hline Age & $0.99(0.97-1.01)$ & 0.169 & - & - \\
\hline Female sex & $0.93(0.53-1.63)$ & 0.798 & - & - \\
\hline Anterior circulation aneurysm & $0.68(0.39-1.20)$ & 0.181 & - & - \\
\hline Length of FU & $1.10(1.00-1.22)$ & 0.051 & - & - \\
\hline Aneurysm diameter & $1.13+(1.07-1.19)$ & $<0.001 \ddagger$ & $1.14(1.08-1.21)$ & $<0.001$ \\
\hline Ruptured aneurysm & $2.06(1.25-3.38)$ & $0.004 \ddagger$ & $2.37(1.39-4.04)$ & 0.001 \\
\hline \multicolumn{5}{|l|}{ Smoking $\mathrm{Hx}$} \\
\hline Never smoker & Reference & NA & Reference & NA \\
\hline Former smoker & $0.78(0.46-1.35)$ & 0.382 & $0.49(0.23-1.05)$ & 0.068 \\
\hline Current smoker & $0.44(0.21-0.91)$ & $0.026 \ddagger$ & $0.82(0.46-1.46)$ & 0.503 \\
\hline \multicolumn{5}{|l|}{ Initial aneurysm occlusion } \\
\hline Residual aneurysm & $1.61(0.70-3.71)$ & 0.263 & - & - \\
\hline Residual neck & $1.58(0.94-2.67)$ & 0.086 & - & - \\
\hline Complete occlusion & Reference & NA & - & - \\
\hline Use of adjunctive device & $0.91(0.50-1.63)$ & 0.740 & - & - \\
\hline
\end{tabular}


A multivariate analysis adjusted for aneurysm rupture status, aneurysm diameter, and aneurysm occlusion indicated that former smokers (OR 0.49, 95\% CI 0.23-1.05; p $=0.07)$ and current smokers (OR $0.82,95 \%$ CI $0.46-1.46$; $\mathrm{p}=0.50$ ) had odds of retreatment similar to those who were never smokers (Table 3). A larger aneurysm diameter was significantly associated with aneurysm retreatment (OR 1.14, 95\% CI 1.08-1.21; p < 0.001), and ruptured aneurysms had a significantly higher rate of retreatment than unruptured aneurysms (OR 2.37, 95\% CI 1.39-4.04; $\mathrm{p}=0.001$ ).

\section{Discussion}

The results of our study indicate that tobacco smoking is not significantly associated with aneurysm recurrence or retreatment among patients undergoing endovascular coiling for intracranial aneurysms. In both uni- and multivariate analyses of the entire cohort, as well as in a subgroup analysis of unruptured aneurysms, we failed to detect an effect of smoking on the risk for either angiographically confirmed recanalization or retreatment. These findings suggest that smoking status should not be a factor for excluding patients for endovascular treatment of intracranial aneurysms. Similar to what has been observed in previous studies, variables associated with a significantly increased rate of aneurysm recurrence included previous rupture, initial aneurysm occlusion, and an increased aneurysm diameter.

Some studies have shown that cigarette smoking is a risk factor for both intracranial aneurysm formation and recurrence after endovascular coiling. It has been proposed that the pathophysiology of aneurysm formation among cigarette smokers results from a decrease in $\alpha 1$-antitrypsin levels., ${ }^{4,5}$ In a study of 110 patients, Ortiz et al. reported an OR of 4.53 for aneurysm recurrence among cigarette smokers when compared with rates of aneurysm recurrence among nonsmokers. ${ }^{11}$ These authors also showed that among patients with SAH, the recurrence rate was positively correlated with SAH severity as measured with the Hunt and Hess scale. We note some important differences between the study by Ortiz and colleagues and the current study. First, the rate of aneurysm recanalization in the Ortiz et al. study was approximately $42 \%$, higher than the $27 \%$ recanalization rate observed in our study. Second, we stratified our patients into 3 groups, smokers, former smokers, and never smokers, whereas Ortiz et al. stratified their patient cohort into never/nonsmokers and former/current smokers. This difference in stratification may be important because the effects of smoking on inflammation markedly diminish after smoking ceases, especially in cardiovascular diseases. ${ }^{1,3}$ In a large study of 607,558 adult patients undergoing major surgery, Musallam et al. observed that only current smoking was associated with poor surgical outcomes and that former smokers had rates of poor outcomes similar to those among nonsmokers. ${ }^{10}$ By stratifying patients into 3 groups, our study could more directly compare the role of perioperative smoking in influencing angiographically determined outcomes of aneurysm treatment.

Our study also provided information on the relation- ship between smoking status and aneurysm recurrence requiring retreatment. A better understanding of the risk factors associated with aneurysm retreatment is important because of the additional costs and risks associated with aneurysm retreatment, although we note that aneurysm recurrence not requiring retreatment is generally associated with low morbidity. Although the results in our study indicated no significant association between smoking and aneurysm recurrence, it is still important to counsel all patients, especially intracranial aneurysm patients, about the risks of smoking.

Our study has several limitations. The patients in our study were stratified into those who never, currently, or formerly smoked, but we did not further stratify smokers by pack-year because such data were not available. Previous studies have shown that increased pack-years are associated with an increased risk of an SAH. Furthermore, we categorized the patients according to smoking status through a retrospective chart review rather than a questionnaire. Because our study was retrospective, we did not perform a power calculation before data collection. Therefore, we were unable to perform a post hoc power calculation to validate the nonsignificant associations between smoking status and aneurysm recurrence or aneurysm retreatment. Additionally, we did not examine outcomes by the type of coils used in the endovascular treatment. Although the coil type used could be an important factor in the risk of aneurysm recurrence after this treatment, several randomized trials have failed to show a major difference in occlusion rates across different coil types. ${ }^{14}$

We did not examine the relationship between the initial degree of occlusion and the recurrence rate. As mentioned above, previous studies have reported that aneurysms that are completely or almost completely occluded have significantly lower recurrence rates than those that are incompletely occluded. The size of the aneurysm neck is another important factor associated with aneurysm recurrence, but we did not include neck size in the analysis. However, this lack of data on neck size was unlikely to play a major role because aneurysms with neck sizes predictive of unfavorable outcomes were usually treated with surgery or with flow diversion rather than with endosaccular coiling.

\section{Conclusions}

The results of our study show that smoking is not an independent risk factor for aneurysm recurrence and retreatment among patients receiving endovascular treatment for intracranial aneurysms. Aneurysm rupture and a larger diameter were the only significant predictors of recurrence. Nonetheless, patients with intracranial aneurysms should continue to be counseled about the risks of cigarette smoking.

\section{References}

1. Athyros VG, Katsiki N, Doumas M, Karagiannis A, Mikhailidis DP: Effect of tobacco smoking and smoking cessation on plasma lipoproteins and associated major cardiovascular risk factors: a narrative review. Curr Med Res Opin 29:1263-1274, 2013

2. Bonita R: Cigarette smoking, hypertension and the risk of 
subarachnoid hemorrhage: a population-based case-control study. Stroke 17:831-835, 1986

3. Boué S, De León H, Schlage WK, Peck MJ, Weiler H, Berges A, et al: Cigarette smoke induces molecular responses in respiratory tissues of ApoE $\mathrm{E}^{-/}$mice that are progressively deactivated upon cessation. Toxicology 314:112-124, 2013

4. Gaetani P, Guallini P, Rodriguez y Baena R, Kiersy C, Grazioli V, Tartara F, et al: Inactivation of alpha1-antiproteinase (alpha1-AT) and changes in antioxidants' plasma levels in subarachnoid hemorrhage. J Neurol Sci 152:154-159, 1997

5. Gaetani P, Tartara F, Tancioni F, Klersy C, Forlino A, Baena RR: Activity of alpha 1-antitrypsin and cigarette smoking in subarachnoid haemorrhage from ruptured aneurysm. J Neurol Sci 141:33-38, 1996

6. Juvela S, Hillbom M, Numminen H, Koskinen P: Cigarette smoking and alcohol consumption as risk factors for aneurysmal subarachnoid hemorrhage. Stroke 24:639-646, 1993

7. Kang HS, Han MH, Kwon BJ, Kwon OK, Kim SH: Repeat endovascular treatment in post-embolization recurrent intracranial aneurysms. Neurosurgery 58:60-70, 2006

8. Knekt P, Reunanen A, Aho K, Heliövaara M, Rissanen A, Aromaa A, et al: Risk factors for subarachnoid hemorrhage in a longitudinal population study. J Clin Epidemiol 44:933-939, 1991

9. Li MH, Gao BL, Fang C, Gu BX, Cheng YS, Wang W, et al: Angiographic follow-up of cerebral aneurysms treated with Guglielmi detachable coils: an analysis of 162 cases with 173 aneurysms. AJNR Am J Neuroradiol 27:1107-1112, 2006

10. Musallam KM, Rosendaal FR, Zaatari G, Soweid A, Hoballah JJ, Sfeir PM, et al: Smoking and the risk of mortality and vascular and respiratory events in patients undergoing major surgery. JAMA Surg 148:755-762, 2013
11. Ortiz R, Stefanski M, Rosenwasser R, Veznedaroglu E: Cigarette smoking as a risk factor for recurrence of aneurysms treated by endosaccular occlusion. J Neurosurg 108:672675,2008

12. Petitti DB, Wingerd J: Use of oral contraceptives, cigarette smoking, and risk of subarachnoid haemorrhage. Lancet 2:234-235, 1978

13. Sacco RL, Wolf PA, Bharucha NE, Meeks SL, Kannel WB, Charette LJ, et al: Subarachnoid and intracerebral hemorrhage: natural history, prognosis, and precursive factors in the Framingham Study. Neurology 34:847-854, 1984

14. White PM, Lewis SC, Gholkar A, Sellar RJ, Nahser H, Cognard C, et al: Hydrogel-coated coils versus bare platinum coils for the endovascular treatment of intracranial aneurysms (HELPS): a randomised controlled trial. Lancet 377:1655-1662, 2011

\section{Author Contributions}

Conception and design: Brinjikji, Lingineni, Kallmes. Acquisition of data: Brinjikji, Gu, Ulsh, Koeller. Analysis and interpretation of data: all authors. Drafting the article: Brinjikji. Critically revising the article: Brinjikji, Gu, Lanzino, Cloft, Kallmes. Reviewed submitted version of manuscript: all authors. Approved the final version of the manuscript on behalf of all authors: Brinjikji. Statistical analysis: Lingineni. Administrative/technical/material support: Ulsh, Koeller. Study supervision: Brinjikji, Kallmes.

\section{Correspondence}

Waleed Brinjikji, 200 1st St. SW, Rochester, MN 55905. email: brinjikji.waleed@mayo.edu. 